\title{
FORMULES GÉNIQUES POUR LA COULEUR DE LA TOISON DU MERINOS A VIANDE ALLEMAND ET DU MOUTON AKARAMAN DE TURQUIE
}

\author{
J.-J. LAUVERGNE \\ Département de Génétique animale, \\ Centre national de Recherches zootechniques I. N.R. A., \\ 78350 Jouy en Josas, (France)
}

\section{RÉSUMÉ}

L'analyse de données de croisements entre Mérinos à viande allemand (Merino fleischschaf) et Akaraman blanc de Turquie recueillies par DüzGüNes et al. (Z. Tierz. Züchtungsbiol., 1960, 74 , 36-47), a été reprise à la lumière de connaissances récemment acquises en matière de génétique de la coloration du mouton. On pense pouvoir préciser la formule de ces deux races vis-à-vis de 4 loci de coloration : Agouti $(A)$, Extension $(E)$, Panachure $S(S)$, Panachure $L(L)$.

- Au locus $A$ gouti $(A)$ on ne trouverait dans les deux races qu'un allele : $A^{\text {wh }}$, qui donne le rouge en présence de l'allèle normal $E^{+}$au locus d'Extension.

- Au locus Extension $(E)$ on trouverait chez l'Akaraman soit l'allèle normal $E^{+}$déjà cité, soit l'allèle $E^{d}$ dominant qui est, en même temps, épistatique sur le rouge au locus $A$ gouti et qui donne du noir. Le Mérinos serait homozygote pour $\mathrm{E}^{+}$.

La fréquence de $E^{+}$dans la race Akaraman blanche aurait atteint une valeur d'équilibre d'environ 0,185 , ce qui correspond à $3,4 \mathrm{p}$. Ioo de brebis taché rouge pour $96,6 \mathrm{p}$. Ioo de brebis taché noir.

- Au locus $S$ de Panachure $S$, on trouverait, chez le Mérinos, l'allele $S^{b}$ qui, sur fond rouge, serait responsable de la couleur blanche de cette race. L'A karaman porterait l'allèle non muté $S^{+}$.

- Au nouveau locus $L$ de Panachure $L$ on trouverait, en race Akaraman blanche, l'allèle muté auquel nous avons donné, provisoirement, le symbole $L^{a k}$ (ak pour Akaraman). Cet allèle serait responsable de la dépigmentation quasi totale de la race, à l'exception de taches centrifuges sur la tête et les pattes. Le gène $L^{a k}$ semble se comporter au moins partiellement comme un dominant et son expressivité, comme celle de $S^{b}$, serait susceptible d'être modifiée par sélection. Avec la différence, toutefois, que la dépigmentation ne peut jamais être totale et que le dessin coloré subsistant est le même, que la mélanine soit rouge ou noire.

Dans ces conditions la formule du Mérinos à viande allemand pourrait s'écrire :

$$
A^{w h} A^{w h} E^{+} E^{+} S^{b} S^{b} L^{+} L^{+}
$$

Elle serait analogue à celle du Berrichon $d u$ Cher.

La formule de l'Akaraman blanc taché de rouge serait alors :

$$
A^{w h} A^{w h} E^{+} E^{+} S^{+} S^{+} L^{a k} L^{a k}
$$


Celle de l'Aharaman blanc taché de noir :

ou

$$
A^{w h} A^{w h} E^{d} E^{+} S^{+} S^{+} L^{a k} L^{a k}
$$

$$
A^{w h} A^{w h} E^{d} E^{d} S^{+} S^{+} L^{a k} L^{a k}
$$

Pour l'Akaraman rouge on peut proposer la formule

$$
A^{w h} A^{w h} E^{+} E^{+} S^{+} S^{+} L^{+} L^{+}
$$

qui est également celle du Solognot français.

Le gène de panachure blanche $L^{a k}$ semble aussi caractériser d'autres races ovines dans le bassin méditerranéen. Il pourrait avoir joué, dans ces régions, un rôle analogue à celui de $S^{b}$ en Europe occidentale pour l'obtention de toisons blanches ou quasiment blanches. Ce désir, sans doute déjà assez ancien, d'avoir des laines blanches répond à un besoin technologique, il semble avoir eu pour résultat de réduire une variabilité génétique qui a pu, comme en témoignent certaines populations reliques, être jadis beaucoup plus importante chez le mouton.

\section{INTRODUCTION}

Des croisements entre le Mérinos à viande allemand et le mouton Akaraman blanc ont été entrepris en Turquie dès I934, pour améliorer la production de laine et la vitesse de croissance des agneaux.

A partir de données recueillies dans des fermes expérimentales de la province de Konya, en Anatolie centrale, DüzgüNes et al. (r960) ont présenté, il y a une quinzaine d'années, une première étude du déterminisme héréditaire de la couleur de la toison. Ce sujet a été repris depuis par Togay (I965).

A l'aide des connaissances récemment acquises sur la coloration du mouton (AdAlsteinsson, I970 ; LAUVERGNe, 1975) il semble possible de pousser un peu plus loin cette analyse génétique. C'est le but du présent article.

\section{MATÉRIEL ET MÉTHODES}

\section{A. - Les races en présence.}

Les données que nous nous proposons d'examiner sont celles que DüzGüNEs et al. (1960) ont rassemblées. Il s'agit essentiellement de croisements de première génération Mérinos $\times A k a$ raman blanc et de back cross sur le Mérinos.

Le Mérinos à viande allemand (all. : Merino fleischschaf, angl. : German Mutton Merino, German Merino, German Precoce, Merino Mutton) tire son origine du Mérinos Précoce importé de France en Allemagne à partir de $190_{4}$ (Mason, 1969). Il est de couleur blanc uniforme.

La race Akaraman est la principale race ovine utilisée en Turquie (6o p. Ioo de l'effectif) (BILGEMRE, I950; MASON, 1967). C'est une race autochtone à queue grasse utilisée pour la viande, le lait et la laine. On distingue deux variétés : la variété blanche et la variété rouge.

Les brebis Akaraman blanches ne sont pas en réalité entièrement blanches. Elles conservent, sur les pattes et sur la tête, des taches de pigment mélanique qui permettent de les classer en deux catégories : taché noir ou taché rouge (cf. Mason, 1967) (fig. I a). 

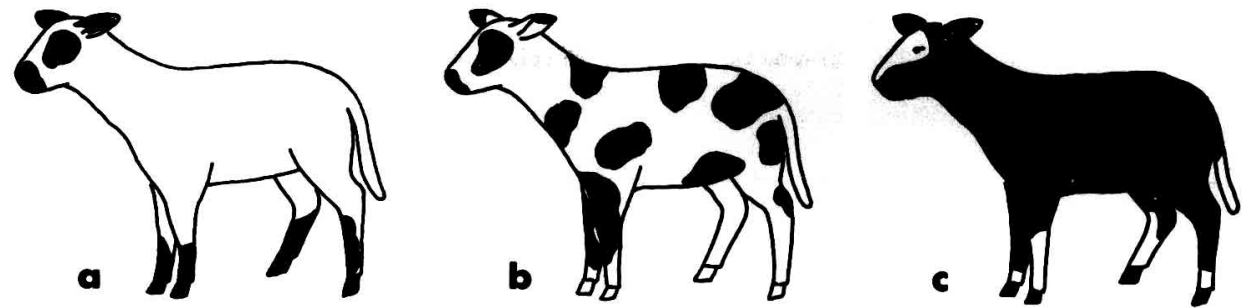

FIG. I. - Differents types de dessins blancs

a) La panachure de l'Akaraman blanc.

Il pourrait s'agir, dans cette race, de l'expression du gène de panachure $L^{\alpha} \boldsymbol{k}$ à l'état homozygote après une sélection pour augmenter l'extension des plages blanches. La pigmentation rémanente peut être noire ou rouge. D'après photos et descriptions de BILGEMre (1950) et Mason (r967).

b) Panachure irrégulière observée sur fond noir en $\mathrm{F}_{1}$ Mérinos $\times$ Akaraman blanc.

Il pourrait s'agir de l'expression du gène de panachure $L^{a k}$ à l'état hétérozygote, en éventuelle interaction avec $S^{b}$ provenant du Mérinos et lui aussi à l'état hétérozygote. D'après la figure I de DüzGüNES et al. (rg6o).

c) Le dessin "points blancs " causé par le gène $S^{b}$ sur fond pigmenté noir : le patron Bizet.

Lorsque la potentialité pigmentaire est le rouge, ce même gène donne un dessin blanc différent, plus irrégulier et plus étendu qui, par sélection, peut même faire disparaître toute présence mélanique dans la toison. Cette interaction entre facteurs suivie de sélection serait, en particulier, responsable de la couleur blanche du Merinos.

\section{Different types of white patterns}

a) Piebaldness in the White Karaman.

May be the expression of the piebaldness gene $L^{a k}$ in the homozygous state after selection to increase white spot extension. Pigmentation may be black or red. From photos and description by BILGemRe (1950) and Mason (1967).

b) Irregular piebaldness on black ground in $\mathrm{F}_{1}$ Merino $\times$ White Akaraman.

May be the expression of piebaldness gene $L^{\alpha k}$ in the heterozygous state possibly interacting with Merino $S^{b}$ also in the heterozygous state. From Figure I of Düzgünes et al. (1960).

c) The " white points" pattern caused by the gene $S^{b}$ on black pigmented ground : Bizet pattern.

When pigment potential is red this same gene gives a different, more irregular, extended pattern which, by selection, may even cause all melanin in the fleece to disappear. This interaction between factors followed by selection seems to be responsible, in particular, for the white color of the Merino.

\section{B. - Les hypothèses de départ}

\section{La formule colorée du Mérinos.}

On va supposer qu'elle est identique à celle du Berrichon $d u$ Cher, que nous avons récemment donnée (LAUVERGNe, 1975) :

$$
A^{w h} A w^{w h} E^{+}+S^{b} S^{b}
$$

C'est-à-dire que le blanc de cette race résulterait de l'interaction du facteur de panachure blanche $S^{b}$ à l'état homozygote. Ce facteur, en présence de la combinaison génique pour le rouge ( $A$ wh au locus $A$ gouti et $E+E+$ au locus d'Extension) donne du blanc total. Cela ne serait pas le cas si le fond pigmentaire était noir.

La raison qui nous incite à adopter pour le Mérinos allemand la même formule que pour le Berrichon est que ce dernier compte parmi ses ancêtres selon QuITTET (I965) les mêmes Mérinos espagnols importés en France au xvirIe siècle qui ont donné les souches de Mérinos précoce, bases du Mérinos à viande allemand comme nous venons de le voir plus haut.

\section{La formule colorée de l'Akaraman blanc.}

La variété Akaraman blanche taché rouge porte probablement tout d'abord comme le Mérinos, $A^{w h}$, au locus $A g o u t i$ et $E^{+}$au locus d'Extension. C'est la seule combinaison factorielle connue pour le rouge chez le mouton (AdALsteinsson, I970; LaUvergne, 1975). Comme nous l'avons vu plus haut la panachure blanche n'est toutefois jamais totale ce qui nous incite à écarter de la formule raciale la présence du mutant $S^{b}$ qui, lui, aurait forcément donné au moins quelques individus tout blanc. L'hypothèse la plus simple est alors du postuler chez l'A présence d'un mutant de panachure à un autre locus que $S$. Nous proposons le symbole $L^{a k}$ pour ce mutant. La formule de l'Akaraman taché rouge pourrait alors s'écrire : 


$$
A^{w h} A^{w h} E^{+} E^{+} S^{+} S^{+} L^{a k} L^{a k}
$$

L'Akaraman blanc taché noir peut tirer, quant à lui, sa couleur noire soit de la formule $E^{d}$ (quels que soient les allèles présents au locus $A$ gouti), on parle alors de noir dominant, soit de la formule $\mathrm{E}+\mathrm{E}+a a$ (noir dit récessif), cf. LAUvergne (I975).

Dans le cas qui nous intéresse il semble que l'on ait affaire au noir de type dominant. Les croisements Akaraman taché noir par Mérinos donnent en effet une majorité de noirs, ce qui ne serait pas le cas s'ils portaient le noir récessif.

La formule proposée de la variété taché noir pourrait donc s'écrire :

$$
\left.A^{w h} A^{w h} E^{d} E^{d} \text { (ou } E^{d} E^{+}\right) L^{a k} L^{a k}
$$

\section{C. - Les ségrégations attendues avec les hypothèses proposées}

I. En $F_{1}$.

a) $F_{1}$ Mérinos $\times$ Akaraman taché rouge.

Elle aurait pour formule $A^{\text {wh }} A^{w h} E^{+} E^{+} S^{b} S^{+} L^{a k} L^{+}$. C'est-à-dire que la couleur pigmentaire serait le rouge et que l'on observerait des dessins blancs produits par l'action conjuguée des deux mutants de panachure à l'état hétérozygote en $S: S^{b} S^{+}$et en $L: L^{a k} L^{+}$.

b) $F_{1}$ Mérinos $\times$ Akaraman taché noir.

La formule serait soit $A$ wh $A$ wh $E+E+S^{b} S+L a k L+$ (les animaux seraient alors pie rouge, comme nous venons de le voir) soit $A^{w h} A^{w h} E^{d} E^{+} S^{b} S^{+} L^{a k} L^{+}$, ce qui donnerait des animaux pie noir avec un dessin blanc sans doute différent de celui que l'on observe sur les $\mathrm{F}_{1}$ pie rouge car l'on sait que $S^{b} n^{\prime} a$ pas le même comportement sur fond noir (donnant le dessin point blanc : liste en tête, extrémité des pattes et de la queue blanches) que sur fond rouge (panachure irrégulière et, le cas échéant, blanc total).

La proportion des $\mathrm{F}_{1}$ pie rouge et des $\mathrm{F}_{1}$ pie noir dans ce croisement dépendrait de la fréquence $q$ du gène $E^{+}$dans la population Akaraman blanche.

\section{En croisements de retour.}

a) Les back cross Mérinos $\times F_{1}$ taché rouge.

On remarque qu'il n'y aurait que deux loci en ségrégation : les deux loci de panachure $S$ et $L$. L'existence de $50 \mathrm{p}$. I 100 de $S^{b} S^{b}$ devrait correspondre à au moins une moitié d'animaux tout blancs le reste étant taché rouge. Mais, comme on l'a vu dans les back cross Berrichon $\times($ Berrichon $\times$ Solognot) LAUVERGNe (1969), il est possible que certains hétérozygotes $S^{b} S^{+}$soient déjà tout blancs, surtout que l'action de $L^{a k}$ à l'état hétérozygote peut augmenter la surface dépigmentée. Dans un premier temps toutefois se contentera de tester la répartition I : I entre les blancs et les pie. rouge.

b) Les back cross Mérinos $\times F_{\mathbf{1}}$ taché noir.

Il y aurait cette fois trois loci en ségrégation : $E, S$ et $L$. Les proportions attendues dans le back cross sont données dans le tableau 1 .

Dans ce tableau on a également mentionné les colorations que pourraient prendre les différents génotypes attendus en se basant sur les connaissances déjà acquises : la formule $S^{b} S^{b}$ donne du tout blanc sur fond rouge et le dessin points blancs sur fond noir; la formule $S^{b} S^{+}$donne une panachure irrégulière sur fond rouge et le dessin point blanc sur fond noir, avec une pénétrance qui peut être de l'ordre de 50 p. Ioo, LAUvergne (1975). Il y aurait aussi un possible comportement dominant, au moins partiel, déjà ci-avant évoqué, du gène $L a k$, qui pourrait, en outre, interréagir avec $S^{b}$ pour donner un dessin blanc différent de la simple juxtaposition des deux dessins. En. simplifiant quelque peu, car les animaux pie ne sont pas décrits avec une grande précision dans l'article des auteurs turcs, on peut tester les proportions $I ; 1 ; 2$ pour, respectivement : les blancs, les pie rouge et les noirs regroupés avec les pie noir.

\section{D. - L'estimation de la fréquence $\mathrm{q}$ de $\mathrm{E}^{+}$chez l'Akaraman}

Au cas où les hypothèses mendéliennes ci-avant proposées seraient acceptables on peut estimer $q$ la fréquence de $E^{+}$de deux façons : raman.

- à partir des proportions des femelles taché rouge et taché noir dans la population $A k a$ -

- à partir des proportions des agneaux taché rouge et taché noir dans le croisement Mérinos $\times$ Akaraman taché noir. 
TABIEAU I

Génotypes ségrégant dans le backcross Mérinos $\times F 1$ taché noir selon les formules géniques proposées pour le Mérinos et l'Akaraman

Segragating genotypes in Merinos $\times$ black Spotted $F_{1}$, according to the proposed genic formula for Merino and Akaraman

\begin{tabular}{|c|c|c|c|c|}
\hline \multirow{2}{*}{ No } & \multicolumn{2}{|c|}{ Formules des gamètes } & \multirow{2}{*}{$\begin{array}{c}\text { Génotypes } \\
\text { des back-cross }\end{array}$} & \multirow[b]{2}{*}{ Colorations prévisibles } \\
\hline & $\begin{array}{c}\text { en provenance } \\
\text { des } F_{1}\end{array}$ & $\mid \begin{array}{c}\text { en provenance } \\
\text { du Mérinos }\end{array}$ & & \\
\hline 1 & $E^{+} S^{+} L^{+}$ & $E^{+} S^{b} L^{+}$ & $E^{+} E^{+} S^{+} S^{b} L^{+} L^{+}$ & $\begin{array}{l}\text { Pie rouge, comme les } \mathrm{F}_{1} \text { Berrichon } \times \text { So- } \\
\text { lognot. }\end{array}$ \\
\hline$\underline{2}$ & $E^{+} S^{+} L^{a k}$ & - & $E^{+} E^{+} S^{b} S^{b} L^{+} L^{a k}$ & $\begin{array}{l}\text { Pie rouge, même formule que les } F_{1} \\
\text { Mérinos } \times \text { Akaraman tachê rouge. }\end{array}$ \\
\hline 3 & $E^{+} S^{b} L^{+}$ & - & $E^{+} E^{+} S^{b} S^{b} L^{+} L^{+}$ & Blanc, même formule que le Mérinos. \\
\hline ' & $E^{+} S^{b} L^{a k}$ & - & $E^{+} E^{+} S^{b} S^{b} L^{+} L^{a k}$ & Blanc, même formule que le Mérinos. \\
\hline 5 & $E^{a} S^{+} L^{+}$ & - & $E^{d} E^{+} S^{+} S^{b} L^{+} L^{+}$ & $\begin{array}{l}\text { Noir à points blancs ou tout noirs, avec } \\
\text { des proportions variables selon la péné- } \\
\text { trance. }\end{array}$ \\
\hline 6 & $E^{a} S^{+} L^{a k}$ & - & $E^{a} E^{+} S^{+} S^{b} L^{a k} L^{+}$ & $\begin{array}{l}\text { Comme en } 5 \text { avec, éventuellement, un } \\
\text { dessin blanc dû à } L^{a k} \text {, si celui-ci est } \\
\text { dominant. }\end{array}$ \\
\hline 7 & $E^{d} S^{b} L^{+}$ & - & $E^{d} E^{+} S^{b} S^{b} L^{+} L^{+}$ & Noir à point blanc, comme le Bizet. \\
\hline 8 & $E^{d} S^{b} L^{a k}$ & - & $E^{d} E^{+} S^{b} S^{b} L^{a k} L^{+}$ & $\begin{array}{l}\text { Noir à points blancs avec, en plus éven- } \\
\text { tuellement un dessin blanc dû à } L^{a k} \text {, } \\
\text { si celui-ci est dominant. }\end{array}$ \\
\hline
\end{tabular}

I. Estimation de q à partir de la population des brebis.

En fait on ne connaît pas les effectifs des brebis elles-mêmes mais ceux des agneaux qu'elles ont donnés, ce qui peut y suppléer, en supposant que la fécondité est la même chez les femelles taché rouge et chez les femelles taché noir. Soit $n_{1}$ le nombre des agneaux nés de mères taché rouge et $n_{2}$ le nombre des agneaux des mères taché noir. En supposant la panmixie et l'équilibre de la fréquence génique réalisés, la fréquence des brebis rouges est $q^{2}$, dont l'estimation par le maximum de vraisemblance est $\frac{n_{1}}{n_{1}+n_{2}}$. On en tire pour $q$ l'estimation $q^{\prime}=\sqrt{\frac{n_{1}}{n_{1}}+n_{2}}$ dont la variance, obtenue par des formules asymptotiques est $\sigma_{q^{\prime}}^{2}=\frac{\mathrm{I}}{4\left(n_{1}+n_{2}\right)}$.

2. Estimation de $\mathrm{q}$ à partir de la fréquence des agneaux dans le croisement Mérinos $\times$ Akaraman taché noir.

Dans les hypothèses de panmixie et d'équilibre de la fréquence génique réalisée la fréquence du gène $E^{+}$dans la fraction de la population femelle taché noir est $\frac{q}{\mathrm{I}+q}$.

Cette valeur est également la fréquence des agneaux rouges que l'on doit observer dans le croisement Mérinos $\times$ Akaraman taché noir, les Mérinos étant $E^{+} E^{+}$.

Si $n_{3}$ est le nombre d'agneaux rouges observés, $n_{4}$ étant celui des agneaux noirs, l'estimation de $\frac{q}{\mathrm{I}+q}$ par le maximum de vraisemblance est $\frac{n_{3}}{n_{4}}$ d'où l'on tire, pour $q$, l'estimation $q^{\prime \prime}=\frac{n_{3}}{n_{4}}$ dont la variance, obtenue en utilisant des formules asymptotiques, est $\sigma_{q^{\prime \prime}}^{2}=\frac{n_{3}\left(n_{3}+n_{4}\right)}{n_{4}{ }^{3}}$. 


\section{RÉSULTATS}

\section{A. - La mise à l'épreuve de nos hypothèses à partir des données turques}

Dans le tableau 2 sont présentées les données rassemblées par DüZGüNEs $e t$ al. (I960) en même temps que les valeurs calculées dans nos hypothèses. L'ajustement y est testé par le $\chi^{2}$.

\section{TABLEAU 2}

Les ségrégations observées par DüzgüNEs et al. (I960) dans des croisements Mérinos à viande allemand et Akaraman, avec le test de l'hypothèse nouvellement présentée

Segregations observed by DüzgüNEs et al. (1960) in crosses between

German Mutton Merino and Akaraman, with the test of newly presented hypothesis

\begin{tabular}{|c|c|c|c|c|c|c|c|c|c|c|c|}
\hline \multicolumn{2}{|c|}{ Croisements } & \multicolumn{2}{|c|}{ Parents } & \multicolumn{7}{|c|}{ Effectif des descendants } & \multirow{3}{*}{$\chi^{2}$} \\
\hline \multirow{2}{*}{ No } & \multirow{2}{*}{ type } & \multirow{2}{*}{ père } & \multirow{2}{*}{ mère } & \multicolumn{2}{|c|}{ taché rouge } & \multicolumn{2}{|c|}{ blanc } & \multicolumn{2}{|c|}{ taché noir } & \multirow{2}{*}{$\begin{array}{l}\text { total } \\
\text { ligne }\end{array}$} & \\
\hline & & & & obs. & calc. & obs. & calc. & obs. & calc. & & \\
\hline 1 & $\mathbf{F}_{1}$ & Mérinos & $\begin{array}{l}\text { Akaraman } \\
\text { taché rouge }\end{array}$ & 7 & & 1 & & 0 & & 8 & \\
\hline 2 & $F_{1}$ & Mérinos & $\begin{array}{l}\text { Akaraman } \\
\text { taché noir }\end{array}$ & 25 & & 6 & & 176 & & 207 & \\
\hline 3 & BC & Mérinos & $F_{1}$ taché rouge & 10 & 9 & 8 & 9 & 0 & & 18 & $0,222 \mathrm{NS}(\mathbf{1})$ \\
\hline 4 & $\mathrm{BC}$ & Mérinos & $\mathrm{F}_{1}$ tout blanc & 0 & & 2 & & & & & \\
\hline 5 & $\mathbf{B C}$ & Mérinos & $F_{1}$ taché noir & 16 & 16,25 & 16 & 16,25 & 33 & 32,5 & 65 & $0,046 \mathrm{NS}\left({ }^{1}\right)$ \\
\hline
\end{tabular}

(1) NS : non significatif au seuil 0,01 .

\section{B. - L'estimation de la fréquence $q$ de $E^{+}$}

Les tests statistiques du tableau 2 ne contredisent pas les hypothèses présentées en ce qui concerne le locus $E$. On peut donc estimer $q$ avec les deux manières proposées ci-avant.

Les valeurs de $n_{1} n_{2} n_{3}$ et $n_{4}$ tirées du tableau 2 sont respectivement $8,207,3$ I et $\mathrm{I} 76$ (en regroupant les blancs avec les taché rouge) on a alors les deux estimées de $q$ :

- $q^{\prime}=0, \mathbf{I} 93$, à partir de la fréquence des brebis taché rouge dans la population, avec $\sigma_{q^{\prime}}=0,034$.

$-q^{\prime \prime}=0,176$, à partir de la fréquence des agneaux taché rouge dans le $F_{1}$ Mérinos $\times$ Akaraman taché noir avec $\sigma_{q^{\prime \prime}}=0,037$. 


\section{DISCUSSION}

\section{A. - Les mutants aux loci $A, E$ et $S$}

Les hypothèses de départ concernant les trois loci $A, E$ et $S$ sont à peu près vérifiées (tabl. 2). On remarque que 1'expressivité de $S^{b}$ à l'état hétérozygote est limitée à la panachure (pas de blanc total) mais nous avions envisagé la possibilité de ce comportement qui s'expliquerait par l'absence, chez l'Akaraman, des modificateurs pour développer l'extension de la dépigmentation causée par $S^{b}$.

\section{B. - L'existence du gène Lak}

Enn fait, à l'extrême limite, on pourrait considérer que ce gène n'existe pas et que la dépigmentation chez l'Akaraman serait due à l'action de plusieurs gènes modificateurs capables de faire varier 1'expressivité de $S^{b}$, qui serait à l'état homozygote dans les 2 races.

Les auteurs turcs n'ont-ils pas, en effet, trouvé une corrélation significative parent/enfant pour l'extension du blanc (DüzGüNEs et al., I960, tab1. 5).

Par ailleurs, un auteur comme ADALSTEINSSON (1970) a pu montrer qu'en race Islandaise, sur des animaux $S^{b} S^{b}$ la sélection pouvait permettre d'obtenir le type de panachure blanche que l'on voulait. Donc, a priori, il ne serait pas impossible d'obtenir de cette manière le dessin centrifuge typique de l'Akaraman (fig. I, a).

Un certain nombre de raisons plaident cependant en faveur de l'hypothèse qui fait intervenir $L^{a k}$ :

- Tout d'abord on voit mal comment, dans les conditions ancestrales de la sélection en Turquie, on ait voulu et pu sélectionner si précisément un tel dessin centrifuge alors que, manifestement, on s'efforçait d'obtenir des animaux le plus blanc possible. Si le gène $S^{b}$ avait existé en présence du gène pour le rouge qui, lui, existe, il est probable qu'on aurait abouti à un animal tout blanc.

- On voit mal en outre pourquoi le gène $S^{b}$ dont le comportement est si différent selon le fond pigmenté en Europe occidentale aurait, en Turquie, acquis un comportement identique en présence du noir et du rouge.

- Enfin, toujours en restant dans 1'hypothèse où le dessin de 1'Akaraman est dû à la présence de $S^{b}$ conjuguée avec $1^{\prime}$ action de modificateurs comment expliquer que, dans tout le bassin méditerranéen, il existe une série de races présentant, sur fond rouge ou noir, le même dessin que l'Akaraman. En se bornant à recenser les illustrations données par MASON (1967) et en ne retenant que les races dont le dessin est bien net (excluant en particulier les dessins pointillés) on note en effet : la race Caussenarde du Lot en France, la race Savoyarde en Italie, la race Churro au Portugal et en Espagne, les races Sjenica et Svrljig, Privor, Dub et Lika en Yougoslavie, Skopelos, Kimi, Chios, Kivircik, Karayaka en Grèce, Daglic en Turquie ainsi que la race Cypriote.

Il faudrait donc admettre dans cette hypothèse de sélection quantitative qu'en divers lieux et diverses époques des éleveurs sans guère de contact entre eux, avec des 
possibilités de sélection limitées, se soient néanmoins attachés à obtenir une expressivité identique en partant d'un gène qui, entre les mains d'autres éleveurs donnait avec la même constance un dessin différent ; soit du tout blanc (sur fond rouge) soit du noir à points blancs. Une telle accumulation de faits a une probabilité très faible de s'être réalisée et incite à repousser 1'hypothèse de gènes modificateurs agissant sur l'expressivité de $S^{b}$ pour retenir comme plus probable, au moins provisoirement, celle de $L^{a k}$.

\section{C. - Le comportement du gène Lak}

Les figures I et 2 de DüzGünEs et al. (I960) permettent de distinguer trois types de dessins blancs sur fond noir :

Type I. Le phénotype Akaraman (cf. fig. 2 des auteurs turcs et notre figure I $a$ ).

Type 2. Un phénotype combiné rappelant l'Akaraman par les dessins sur la tête et les pattes mais avec des taches sur le corps (cf. fig. I des auteurs tures et notre figure $\mathbf{I} b$.)

Type 3. Un phénotype qui rappelle le noir à point blanc du Bizet (cf. semble-t-il quelques individus de la figure 2 des auteurs turcs et notre figure I $c$ ).

En admettant que $L^{a k}$ est dominant avec une expressivité plus variable et moins de blanc chez 1'hétérozygote que chez l'homozygote les types I et 3 correspondraient aux génotypes $L^{a k} L^{+}$sur fond noir que nous rencontrons en $F_{1}$ et back-cross sur le noir (cf. tabl. I) alors que le type 3 correspondrait aux génotypes $S^{b} S^{b} L^{+} L^{+}$ou $S^{b} S^{b} L+L+$ toujours sur fond noir.

\section{D. - Les interprétations des auteurs turcs}

DüzGÜNES et al. (I960) postulaient que 1'Akaraman blanc taché noir possédait à la fois un facteur pour le rouge $(B)$ et un autre, $(A)$ qui était épistatique sur $B$. C'està-dire qu'ils avaient pressenti le rôle de 2 loci pour le contrôle de la pigmentation. Toutefois, leur interprétation s'écarte de la nôtre quand ils considèrent que les animaux blancs (Mérinos ou croisés) ne portent ni le facteur $A$, ni le facteur $B$, mais des allèles récessifs $a$ et $b$. C'est-à-dire qu'ils rangent dans les mêmes séries alléliques des gènes contrôlant la pigmentation proprement dite et d'autres contrôlant la panachure blanche.

Or, d'après ce que l'on sait des gènes de couleur des Mammifères (SEARLE, I968) les loci qui contrôlent 1'élaboration du pigment comme Agouti ou même Extension ne contrôlent pas en même temps un dessin blanc donné. Ces plages blanches typiques dans le pelage résultent en effet de l'absence de tout pigment mélanique (Fox et VEVERS, I960). Elles sont déterminées par des allèles qui ont une action purement négative et bien localisée sur la genèse du pigment et sa distribution en altérant la migration du mélanocyte et son comportement, en perturbant le fonctionnement d'un enzyme etc...

L'interprétation donnée plus récemment par ToGAY (1965) fait intervenir des mutants à 4 loci différents. Elle ne nous semble pas marquer de progrès notables par rapport à celle de DüzGǘNEs et al. (I960). Toutefois en l'absence d'une traduction in extenso du texte turc, nous n'avions à notre disposition pour comprendre cet auteur qu'un résumé assez court en anglais. 
Le regroupement allélique abusif si souvent fait à propos de la coloration du mouton est dû, comme nous pensons l'avoir montré, au comportement très particulier du mutant de panachure $S^{b}$ (cf. LAUVERGNE, I975).

\section{E. - La fréquence de $E^{+}$à l'équilibre}

Les deux estimées, $q^{\prime}=0, \mathrm{I} 93 \pm 0,068$ et $q^{\prime \prime}=0, \mathrm{I} 76 \pm 0,074$, de $q$ ne sont pas significativement différentes. On peut penser queles hypothèses de panmixie et d'équilibre génique réalisé sont acceptables. Comme les variances de ces deux estimateurs sont quasi égales on peut prendre comme meilleur estimateur de la fréquence de $E^{+}$ en race Akaraman leur moyenne arithmétique qui est ici $\bar{q}=0, \mathrm{I} 845$.

Il reste à préciser les forces sélectives qui causent cet équilibre. On a vu récemment que $E^{+} E^{d}$ n'avait pas d'avantage sélectif sur $\mathrm{E}^{+} \mathrm{E}^{+}$, au moins en ce qui concerne la fertilité des femelles (LAUVERGNE, I976). Toutefois, en matière de coloration le jeu de la préférence de certains éleveurs pour le taché noir ou le taché rouge peut très bien suffire pour assurer l'équilibre (cf., par exemple, l'influence de l'homme sur la fréquence des gènes de coloration du pelage du chat domestique, CLARK, I975).

\section{F. - La variabilité des mutants colorés en Turquie}

On se rappelle que la race Akaraman avec ses deux variétés : blanches (taché noir ou rouge) et rouge ou violette (qui est simplement probablement de génotype $A{ }^{w h} A{ }^{w h} E^{+} E+S^{+} S+L^{+} L^{+}$, comme en France le Solognot) constitue 60 p. Ioo du cheptel ovin turc. Les autres races autochtones par ailleurs ne semblent pas présenter de phénotypes colorés fort différents de ceux observables chez 1'Akaraman (BILGEMRE, I950, MASON, I967). On est alors frappé du petit nombre de gènes de coloration décelables dans un pays d'élevage à dominante ovine (6o millions de têtes), dont le territoire empiète largement sur la zone considérée comme le berceau de la $\bullet$ domestication du mouton (ISAAC, I970) et où, par conséquent, les mutants auraient dû s'accumuler depuis des millénaires.

On trouverait ainsi dans la population autochtone de Turquie seuls deux loci ségrégant avec deux allèles chacun $\left(E^{d} / E^{+}\right.$et $\left.L^{+} / L^{a k}\right)$.

Toutefois, à la réflexion, il n'y a pas lieu d'être tellement étonné car cette situation se retrouve dans d'autres pays où l'élevage ovin joue, ou a joué un rôle important. Ainsi, en France continentale, on ne trouve que des moutons blancs (en proportion écrasante), des moutons blancs à tête tachetée de rouge et de noir et quelques races noires ou rouges (QUITTET, I965).

L'existence de certaines populations bien plus variées quant à leur coloration et que l'on s'accorde d'ailleurs à considérer, au moins sous cet aspect, comme des reliques (les races Islandaise, Corse, certaines races de Scandinavie et d'Écosse, (LAUVERGNE et ADALSTEINSSON, I976) laisse à penser que cette situation peut être en Europe occidentale, secondaire. Elle résulterait d'une sélection pour obtenir des toisons le moins pigmenté possible, à cause des exigences de la teinture.

En Turquie, on note également la forte prédominance des phénotypes quasiment blancs : 1'Akaraman rouge, seule race pigmentée notable, ne regroupe que ro p. Ioo du cheptel (MASON, I967). Il est probable que les mêmes raisons technologiques qu'en Europe occidentale ont joué pour aboutir à la réduction de la variabilité génique constatée en Turquie. 


\section{CONCLUSION}

La couleur du Mérinos à viande allemand semble résulter de la même combinaison génique que celle du Berrichon $d u$ Cher cependant que 1'étude de 1'Akaraman blanc révèle l'existence probable d'un autre mutant de panachure blanche, provisoirement nommé $L^{a k}$. Ce mutant, qui paraît avoir une certaine extension dans le bassin méditerranéen oriental, pourrait avoir, dans cette zone-là, joué le même rôle que le gène $S^{b}$ en Europe occidentale pour l'obtention de toisons blanches ou quasiment blanches.

Reçu pour publication en mai 1976 .

\section{REMERCIEMENTS}

Une mention spéciale aux auteurs turcs DÜzgüNEs et al. dont nous empruntons les données doit être tout d'abord faite.

Nous voudrions remercier aussi tout particulièrement le $D^{r} \mathrm{~S}$. Adalsternsson (Institut de Recherches Agronomiques, Rejkjavik, Islande) qui a relu avec soin le manuscrit et suggéré de fort utiles remaniements et le professeur G. LEFORT (chaire de Mathématique I.N.A. Paris-Grignon) dont les remarques ont été précieuses pour la discussion statistique.

\section{SUMMARY}

\section{GENIC FORMULAS FOR FLEECE COLOR OF GERMAN MUTTON MERINO AND TURKISH KARAMAN SHEEP}

Analysis of data on crosses between German mutton merino (Merino fleischschaf) and Turkish White Karaman gathered by DüzGüNes et al. (Z. Tierz. Züchtungsbiol., 1960, 74, 36-47) is reviewed in the light of recent knowledge in the field of genetics on coloring of sheep. The formula of these two breeds as related to 4 coloring loci : Agouti (A), Extension (E), Piebaldness $S$ (S), Piebaldness $L(L)$ seems to be determined as :

- Only one allele $\left(A^{w h}\right)$ is found at $A$ gouti locus in both breeds ; it gives red in the presence of normal allele $E^{+}$at the Extension locus.

- Either the normal allele $E^{+}$already mentioned or the dominant allele $E^{d}$ is found at locus Extension $(E)$ in the Karaman. The latter allele is epistatic on the red at $A$ gouti locus and gives black. The Merino would be homozygous for $E^{+}$.

$E^{+}$frequency in the White Akaraman breed would reach an equilibrium value of about 0.185 ; this corresponds to $3.4 \mathrm{p}$. roo of red spotted sheep for $96.6 \mathrm{p}$. roo of black spotted sheep.

- Allele $S^{b}$ is found at locus $S$ of Piebaldness $S$ in the Merino. On red ground this allele could cause the white color of the breed. The Akaraman would carry the non-mutated allele $S^{+}$.

- At the new locus $L$ of Piebaldness $L$ of the White Akaraman breed is found a mutated allele which we provisionally represent by the symbol $L^{a k}$ (ak for Akaraman, the turkish name of the breed). This allele would cause almost complete depigmentation, except for centrifugal spots on the head and legs. The $L^{a k}$ gene seems to be partially dominant on $L^{+}$and its expression, as that of $S^{b}$, could be changed by selection. However, contrary to $S^{b}$, the depigmentation it gives can never be total and the color pattern, is always the same, whether red or black melanin.

In these conditions the formula of the German mutton Merino can be written :

$$
A^{w h} A^{w h} E+E+S^{b} S^{b} L+L+
$$


It is analogous to that of the Berrichon $d u C h e r$.

The formula of the red spotted White Karaman would be :

$$
A{ }^{w h} A{ }^{w h} E+E+S+S+L a k L a k
$$

That of the black spotted White Karaman:

$$
A^{w h} A^{w h} E^{a} E^{+} S+S+L^{a k} L^{a k}
$$

or

$$
A^{w h} A^{w h} E^{a} E^{d} S^{+} S^{+} L^{a k} L^{a k}
$$

The formula :

$$
A{ }^{w h} A{ }^{w h} E^{+} E+S+S+L+L+
$$

may be proposed for the Red Karaman; it is also that of the French Solognot.

The white piebaldness gene $L^{a k}$ also seems to characterize other sheep breeds in the Mediterranean basin. In these regions, it may have played a role analogous to that of the $S^{b}$ in western Europe in the obtainment of white or nearly white fleeces. This preference for white wool, probably of long date, answers a technological need. It seems to have reduced genic variability which once was, as some old populations attest, much greater in sheep.

\section{RÉFÉRENCES BIBI,IOGRAPHIQUES}

Adalsteinsson S., I970. Colour inheritance in Icelandic sheep and relation between colour, fertility and fertilization. J. agr. res. Icel., 2, 3-135.

BILGEMRE K., 1950. Sheep raising in Turkey. 245-273- (sans référence, mais celle-ci pourrait être r950: Relaz. Congr. int. allev. ovino, Roma, 1949).

CLARK J. M., I975. The effects of selection and human preference on coat colour gene frequencies in urban cats. Heredity, 35, r95-210.

Düzg ÜNeS O., YaRkiN, I., Sönmez R., I96o. A study on the variation in colour in the fat-tailed White-Karamen sheep, Merino-Fleisch-Schafe and their crosses. Z. Tierz. Züchtungsbiol., 74, 36-47.

Fox H. M., Vevers H. G., I96o. The nature of animal colours. Sidgwick and Jackson, London.

IsAAC E., I970. Geography of domestication Prentice-Hall, Englewood Cliffs N. J. U.S.A., 80-81.

Lauvergne J. J., I969. Hérédité de la couleur blanche du mouton Berrichon croisé à des Solognots. Ann. Génét. Sêl. anim., 1, 219-226.

LAUVERGNe J. J., 1975. Génétique de la couleur de la toison de trois races ovines françaises : Berrichonne, Bizet et Solognote. Ann. Génét. Sél. anim., $7,263-276$.

Lauvergne J. J., 1976. Fertilité comparée des brebis $E^{+} E^{+}$et $E^{d} E^{+}$. Ann. Génét. Sél. anim., 8 (sous presse).

Lauvergne J. J., Adalsteinsson S., 1976. Gènes pour la coloration de la toison de la brebis Corse. Ann. Génét. Sêl. anim., 8, 153-172.

MAson I. L., I967. The sheep breeds of the Mediterranean., FAO, Rome and Commonwealth Agricultural Bureaux, Farnham Royal, Bucks, England, I35-14I, I83-184.

Mason I. L., I969. A world dictionary of livestock breeds, types and varieties. Commonwealth Agricultural Bureaux, Farnham Royal, Bucks, England (2nd revised ed.), 207.

Quittet E., 1965. Races ovines françaises (2e éd.). La Maison rustique, Paris, 26-29, 90-93.

SEARLE A. G., 1968. Comparative genetics of coat colour in Mammals. Logos Press. London.

Togay H. G., 1965. Merinos $\times$ Akaraman Melezlerinde Renk Kalitimi. Lalahan Zootek. Arastirma Enst. Yagin, (18), pp. 43 (en turc, résumé anglais). 